\title{
The Application of Three Order Cumulants Coupling Features in Fault Diagnosis
}

\author{
Wenbing Wu, Rihua Liu \\ Nanchang Normal University, Nanchang, China \\ Email: wwbysq@fjnu.edu.cn
}

How to cite this paper: $\mathrm{Wu}, \mathrm{W} . \mathrm{B}$. and Liu, R.H. (2020) The Application of Three Order Cumulants Coupling Features in Fault Diagnosis. International Journal of Modern Nonlinear Theory and Application, 9, 81-89.

https://doi.org/10.4236/ijmnta.2020.94006

Received: September 24, 2020

Accepted: November 10, 2020

Published: November 13, 2020

Copyright $\odot 2020$ by author(s) and Scientific Research Publishing Inc. This work is licensed under the Creative Commons Attribution International License (CC BY 4.0).

http://creativecommons.org/licenses/by/4.0/

\begin{abstract}
Although mechanical vibration is extremely complicated, each fault signal produced by it has its own inherent features, The distinction may be most prominent between the certain components hidden in those features and the same components of normal signals. Three-order cumulant can reduce the Gaussian background noise automatically and its complex formal includes different coupling information of its signal. In the experiment, through these different coupling modes, the same coupling components are fetched from specific fault signal and normal signal, then these components are used to diagnose that certain fault. The results show that the method can fetch the most prominent distinction between normal signal and the specific fault signal, so the specific fault diagnosis by this method is satisfactory.
\end{abstract}

\section{Keywords}

Three Order Cumulant, 1 (1/2) Dimension Spectrum, Coupling, Fault Diagnosis

\section{Introduction}

Because the high-order cumulant of the Gaussian process (when the order is greater than 2) is equal to zero, and for non-Gaussian processes, there is at least some order $\mathrm{k}$ greater than 2 , and the $\mathrm{k}$-order cumulant is not equal to zero. Therefore, the use of high-order cumulants can automatically suppress the influence of Gaussian background noise (colored or white), establish a non-Gaussian signal model under Gaussian noise, and extract non-Gaussian signals (including harmonic signals) from Gaussian noise. Because of this, the statistic of high-order cumulants has received increasing attention and has become a very useful tool in signal processing. The bispectrum derived from the third-order cumulant contains the asymmetric and nonlinear information of the signal, which can be used to describe the nonlinear phase coupling, especially the 
second phase coupling, which has been widely used in fault diagnosis [1] [2], but currently the application of higher-order spectra is mostly limited to the real number field. W. R. Raghuveer and domestic scholar Wang Shuxun have conducted in-depth studies on the coupling properties of complex higher-order cumulants, and clearly pointed out the differences of various complex higher-order cumulants. Define the coupling characteristics of the form [3] [4] [5] [6] [7]. Based on this, this paper uses the different coupling characteristics of complex high-order cumulants to carry out fault identification research.

\section{High-Order Cumulants}

Let $\{x(n)\}$ be a zero-mean $k$-order stationary random process, then the $k$-order cumulant $c_{k x}\left(\tau_{1}, \tau_{2}, \cdots, \tau_{k-1}\right)$ of the process is defined as the k-order joint cumulant of the random variable $\left\{x(n), x\left(n+\tau_{1}\right), \cdots, x\left(n+\tau_{k-1}\right)\right\}$, namely

$$
c_{k x}\left(\tau_{1}, \tau_{2}, \cdots, \tau_{k-1}\right)=\operatorname{cum}\left\{x(n), x\left(n+\tau_{1}\right), x\left(n+\tau_{2}\right), \cdots, x\left(n+\tau_{k-1}\right)\right\}
$$

The $k$-th moment $m_{k x}\left(\tau_{1}, \tau_{2}, \cdots, \tau_{k-1}\right)$ of this process is defined as the $k$-th joint moment of the random variable

$$
\left\{x(n), x\left(n+\tau_{1}\right), \cdots, x\left(n+\tau_{k-1}\right)\right\},
$$

namely:

$$
m_{k x}\left(\tau_{1}, \tau_{2}, \cdots, \tau_{k-1}\right)=\operatorname{mom}\left\{x(n), x\left(n+\tau_{1}\right), \cdots, x\left(n+\tau_{k-1}\right)\right\}
$$

Here, $\operatorname{mom}()$ represents the joint moment, and the third-order cumulant is:

$$
c_{3 x}\left(\tau_{1}, \tau_{2}\right)=E\left\{x(n) x\left(n+\tau_{1}\right) x\left(n+\tau_{2}\right)\right\}
$$

The $k$-order cumulant spectrum is defined as the $k-1$ dimensional Fourier transform of the $k$-order cumulant, namely

$$
S_{k x}\left(\omega_{1}, \cdots, \omega_{k-1}\right)=\sum_{\tau_{1}=-\infty}^{\infty} \cdots \sum_{\tau_{k-1}=-\infty}^{\infty} c_{k x}\left(\tau_{1}, \cdots, \tau_{k-1}\right) \exp \left[-j \sum_{i=1}^{k-1} \omega_{i} \tau_{i}\right]
$$

The third-order spectrum (also called bispectrum) is:

$$
B_{x}\left(\omega_{1}, \omega_{2}\right)=\sum_{\tau_{1}=-\infty}^{\infty} \sum_{\tau_{2}=-\infty}^{\infty} c_{3 x}\left(\tau_{1}, \tau_{2}\right) \mathrm{e}^{-j\left(\omega_{1} \tau_{1}+\omega_{2} \tau_{2}\right)}
$$

After the AR model is established, the bispectral expression is:

$$
B^{A R}\left(\omega_{1}, \omega_{2}\right)=\gamma_{a .3} H\left(\omega_{1}\right) H\left(\omega_{2}\right) H^{*}\left(\omega_{1}+\omega_{2}\right)
$$

Let the frequency variable $\omega_{1}=\omega_{2}=\omega$, the diagonal slice of the bispectrum can be obtained as

$$
B S_{1.5 D}(\omega, \omega)=\gamma_{3 a} H^{2}(\omega) H^{*}(2 \omega)
$$

where, $H(\omega)=\frac{1}{1+\sum_{i=1}^{p} \phi_{i} \mathrm{e}^{-j i \omega}}$ is the frequency characteristic function,

$\gamma_{3 a}=E\left\{a^{3}(n)\right\}$ is skewness, $a(n)$ is an independent and identically distributed non-Gaussian stochastic process, $\varphi_{i}$ and $p$ are the coefficient and order of the 
AR model, respectively, $H^{*}(\omega)$ is the conjugate function of $H(\omega)$.

\section{3. $1 \frac{1}{2}$ Dimension Spectrum}

According to literature (6), in formula (3), let $x(n)$ be a complex signal, and define it as follows:

Definition 1:

$$
c_{3 x}\left(\tau_{1}, \tau_{2}\right)=E\left\{x(n) x\left(n+\tau_{1}\right) x\left(n+\tau_{2}\right)\right\}
$$

Definition 2:

$$
c_{3 x}\left(\tau_{1}, \tau_{2}\right)=E\left\{x^{*}(n) x\left(n+\tau_{1}\right) x\left(n+\tau_{2}\right)\right\}
$$

Definition 3:

$$
c_{3 x}\left(\tau_{1}, \tau_{2}\right)=E\left\{x^{*}(n) x\left(n+\tau_{1}\right) x^{*}\left(n+\tau_{2}\right)\right\}
$$

where $x^{*}(n)$ is the conjugate complex number of $x(n)$, and the complex signal in this article is obtained by hilbert transformation of the collected original signal. On this basis, let $\tau_{1}=\tau_{2}=\tau$, 1-D diagonal slice of the third-order cumulant $c_{3 x}(\tau, \tau)$ can be obtained, and then perform Fourier transform on the slice to obtain the $1 \frac{1}{2}$ dimensional spectrum:

$$
B(\omega)=\sum_{\tau=-\infty}^{\infty} c_{3 x}(\tau, \tau) \mathrm{e}^{-j \omega \tau}
$$

Literature (6) pointed out that according to the way of definition 1, the third-order diagonal cumulative of the signal that can be expressed in the form of complex harmonics is 0 , and the third-order diagonal cumulative of the signal that can be expressed in the form of definition 2 only contains the components generated by coupling. The way of defining three only includes the components participating in the coupling.

\section{Data Collection}

In this experiment, 18 sets of data, a total of 72 sets were collected in the normal working state of the overflow valve and the three fault states. The number of data used in each set of data in the experiment is 1024. The filtered signal is shown in Figure 1.

\section{Spectra}

This experiment first combines the normal state and the three fault states. State $0,1,2$ and 3 represent the normal state and the three fault states respectively, and then let $(0,1),(0,2)$ and $(0,3)$ represent the data pairs that require fault identification after the normal state and various fault states are combined. Each data pair contains 36 sets of data. Then, according to the aforementioned three definition methods, the $1(1 / 2)$ dimension Spectrum of each set of data in each data pair is obtained. This paper selects $(0,3)$ data pairs for illustration. When 
the oil pressures are $1 \mathrm{MPa}, 2 \mathrm{MPa}$, and $5 \mathrm{MPa}$, respectively, select $(0,3)$ data pairs for the dimension spectrum obtained under the three definition methods to select 1 set of icons. In Figure 2, Figure 3 and Figure 4, the horizontal axis in the figure represents the frequency of the circle, the unit is, and the vertical axis represents the normalized amplitude, which is dimensionless. It can be seen that under the definition of the three states, the difference between the normal state and the fault state is more obvious in terms of the composition and density of the spectral peaks.

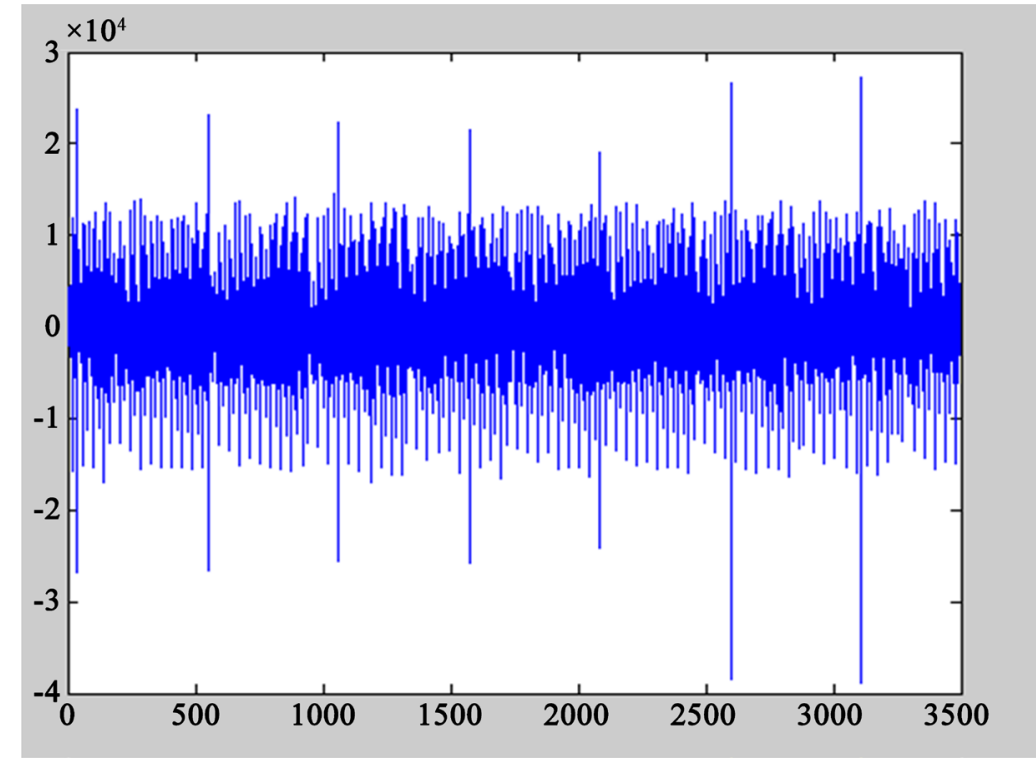

Figure 1. Filtered signal.
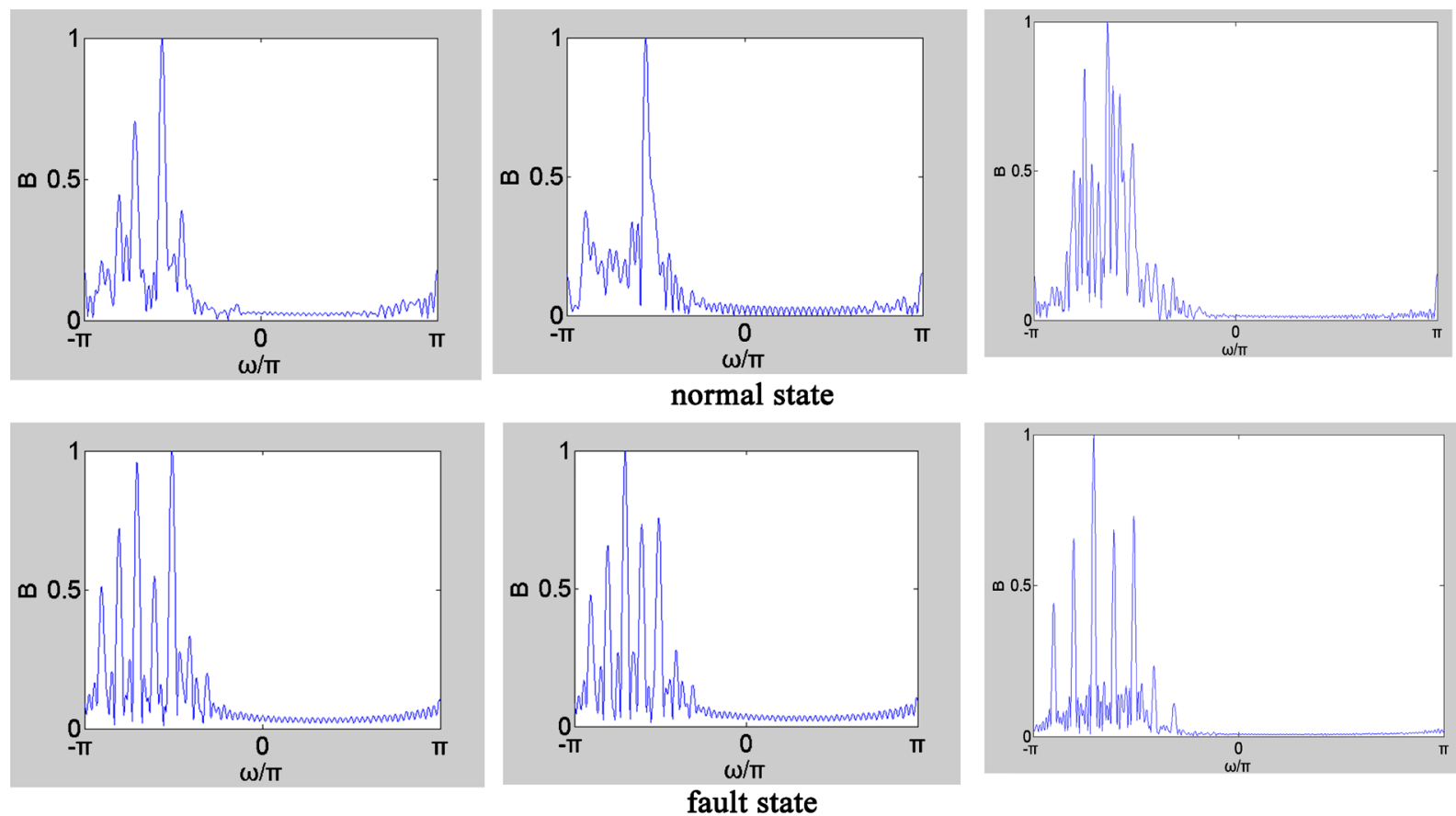

Figure 2. $1(1 / 2)$ dimension spectrum in definition 1. 

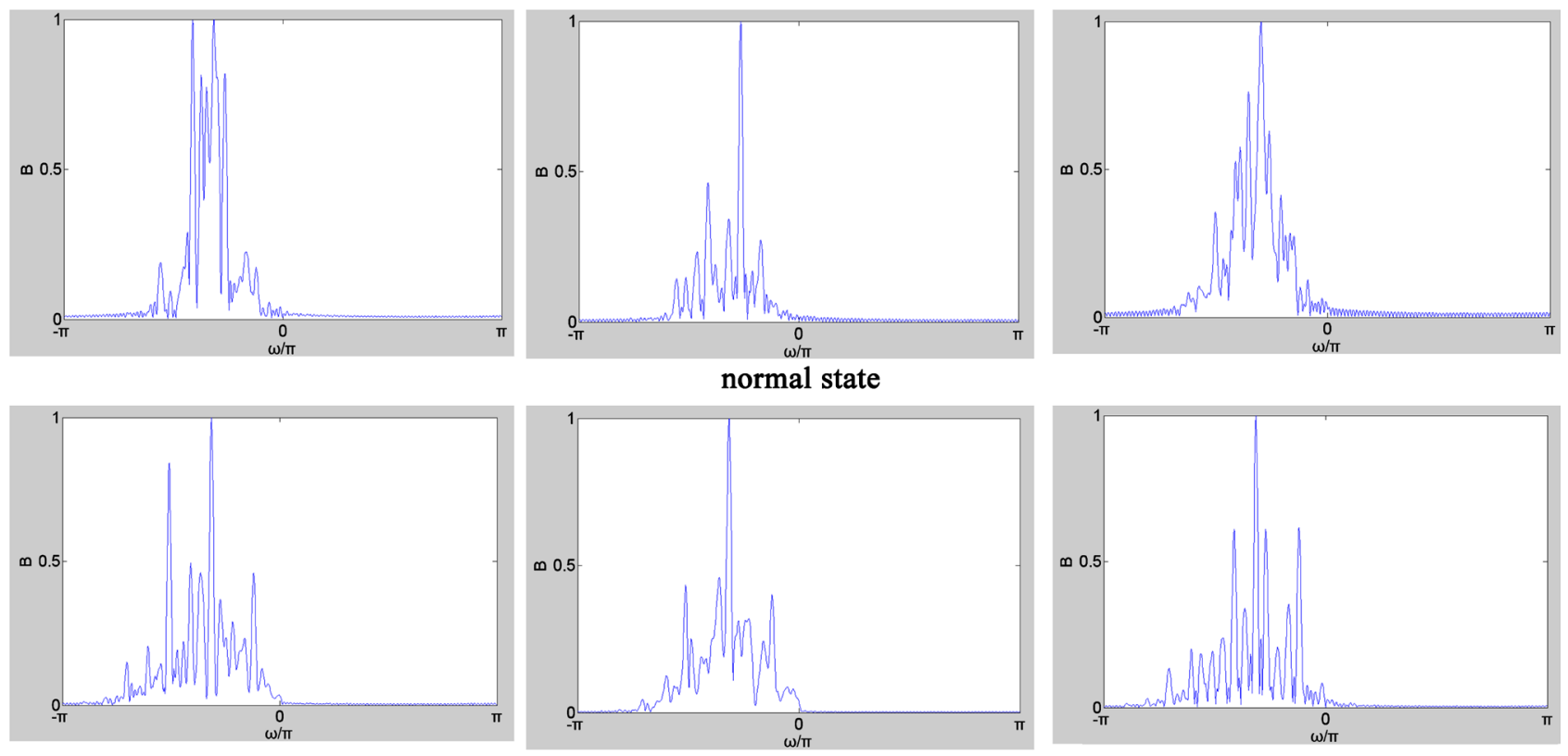

Figure 3. $1(1 / 2)$ dimension spectrum in definition 2.
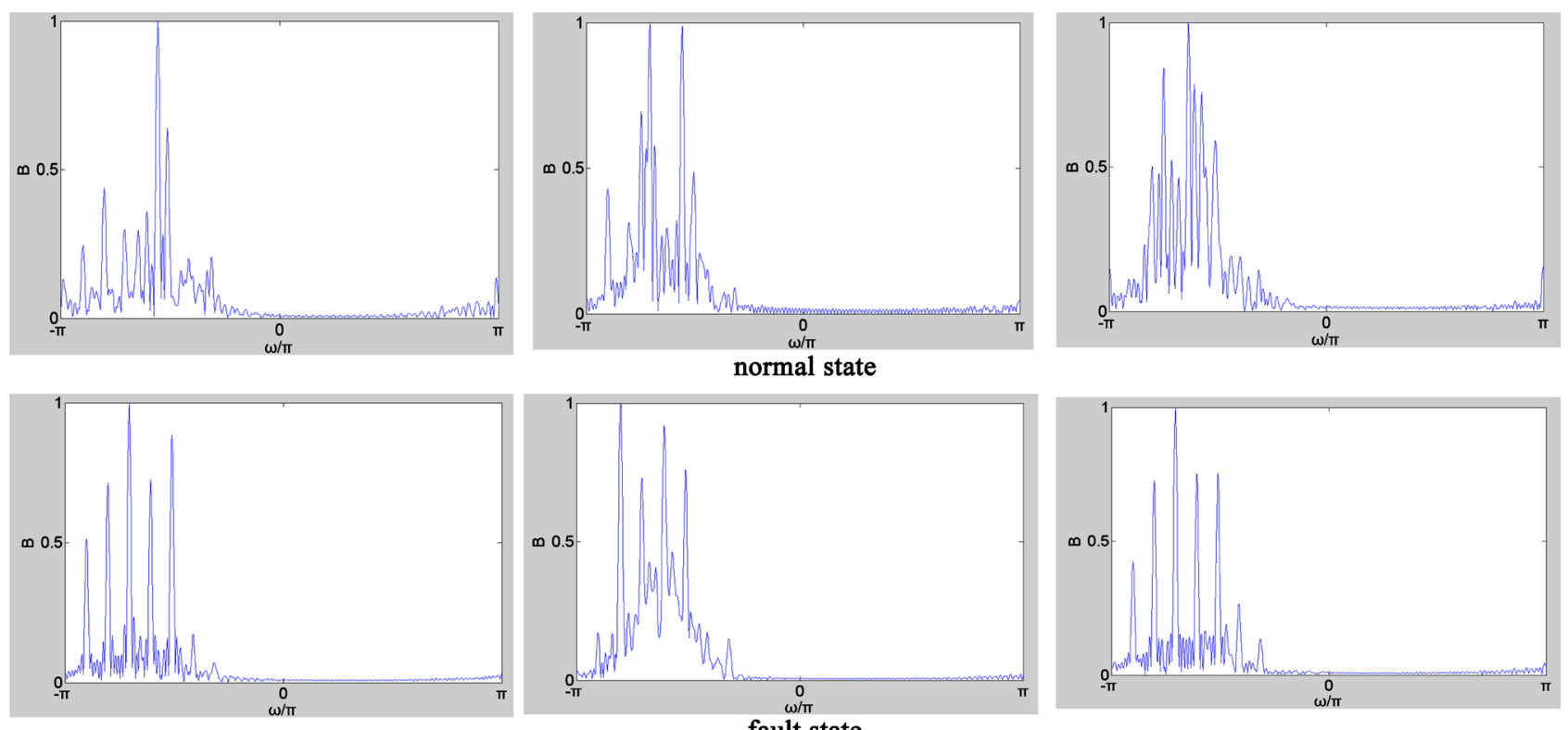

Figure 4. $1(1 / 2)$ dimension spectrum in definition 3.

\section{Experimental Results}

In order to effectively identify faults, wavelet packets are used as tools. The 8 wavelet reconstruction coefficients obtained after the signal is decomposed by the three-layer wavelet packet are used as the feature vector, then they were Input into the support vector machine for fault diagnosis. Table 1 shows the eigenvectors obtained by the $(0,3)$ data pair in the definition 1 mode.

In this paper, the same LSSVM is established based on the literature (10). For each type of data pair, take the three definition methods under $(0,3)$ data pair as 
an example. When training LSSVM, the normal state and the fault state are coded as 1 and -1 respectively, because the normal data and the fault data are obtained under 5 different oil pressures. When training, take out the corresponding 5 sets of data from the normal data and fault data in Table 1 and a total of 10 sets, and train the established least square support vector machine. After training, the support vector machine is used for fault identification, and the training network is used to simulate and identify the remaining 26 sets of data. As shown in Figure 5, the figure shows the simulated state of the 26 sets of data. The circle indicates the preset state of the training data. For example, the first 13 sets of data are measured normal data and should be in the state 1 represented by the upper horizontal line in the figure, and the next 13 sets of fault data should be in the next horizontal line-1 state, the red solid circle represents the classification result of the data after the LSSVM simulation. If the hollow circle of a certain group of data overlaps with the red solid circle, it means that the simulation result of this group of data is correct, otherwise it is wrong. The simulation result is shown in Figure 5. The result shows that in the case of $(0,3)$ data

Table 1. The eigenvector of fault 3 of definition 1.

\begin{tabular}{lllllllll}
\hline normal state & 0.5803 & 0.1809 & 0.0777 & 0.0620 & 0.0379 & 0.0280 & 0.0139 & 0.0194 \\
& 0.5731 & 0.1722 & 0.0725 & 0.0740 & 0.0357 & 0.0323 & 0.0156 & 0.0246 \\
& 0.6126 & 0.1528 & 0.0681 & 0.0695 & 0.0328 & 0.0329 & 0.0135 & 0.0177 \\
& & & \multicolumn{7}{c}{$\ldots \ldots$} \\
fault 3 state & 0.5736 & 0.1758 & 0.0728 & 0.0658 & 0.0346 & 0.0298 & 0.0191 & 0.0284 \\
& 0.5832 & 0.1721 & 0.0733 & 0.0671 & 0.0350 & 0.0306 & 0.0156 & 0.0230 \\
& 0.6344 & 0.1464 & 0.0572 & 0.0669 & 0.0265 & 0.0311 & 0.0144 & 0.0231
\end{tabular}

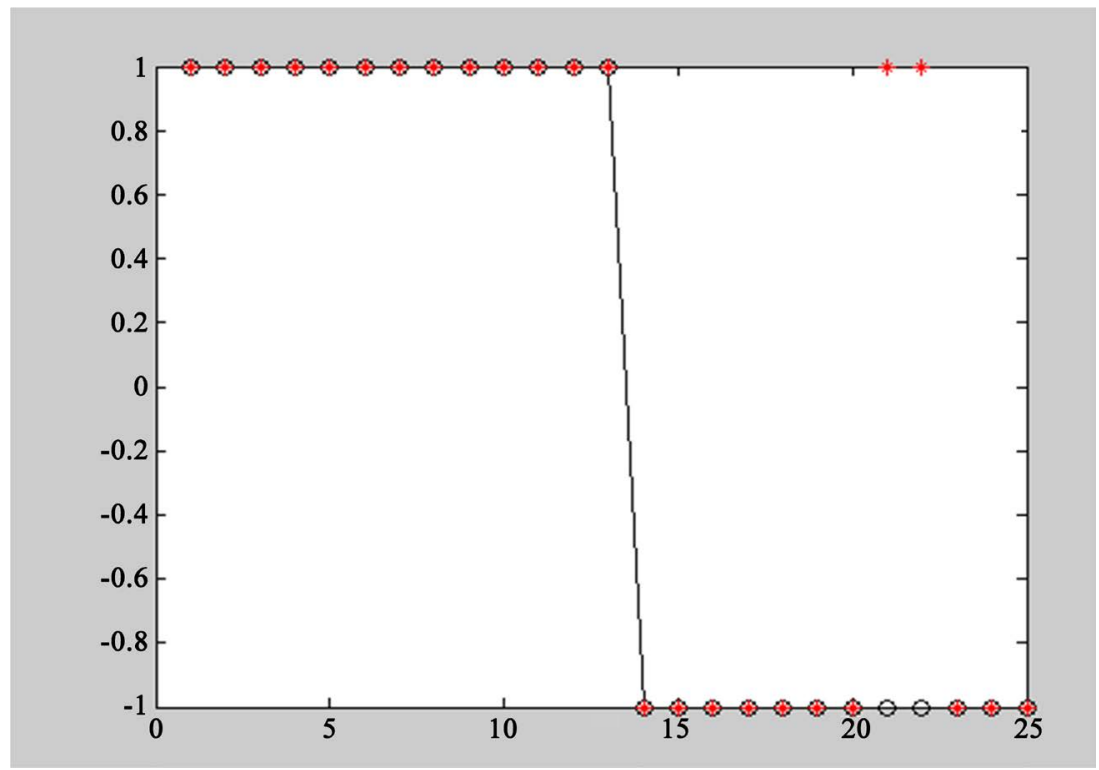

Figure 5. Definition 3 and fault 3 simulation results. 
pair, the recognition is carried out in the way of definition 3, and there are only 2 groups of recognition errors. In order to facilitate the comparison, the experimental results of the three different definition methods under the three failure states are shown in Table 2. The numbers in the table represent the number of errors in the simulation recognition. For further analysis, the same experiment was performed on the diagonal slices of the bispectrum, and the results are also shown in Table 2. Figure 6 shows the result of simulated recognition in the case of $(0,3)$ data pairs in the form of bispectral diagonal slices.

\section{Analysis of Experimental Results}

It can be seen from Table 2 that in the third fault state, there are only 2 groups of identification errors when the fault is identified according to the method of definition 3, and there are only 3 groups of errors in the method of definition 2 at the time of fault 2. As mentioned earlier, the third-order diagonal cumulant in the way of definition 2 only includes the components generated by the coupling, while the way of defining three only includes the components participating in the coupling. It can be inferred from these experimental results that although each type of failure occurs. The mechanism is different. For example, the third fault in this experiment is due to the artificial addition of iron cores at the inlet

Table 2. Number of simulation result errors.

\begin{tabular}{cccc}
\hline Kinds of fault & Fault $\mathbf{1}(\mathbf{0 , 1 )}$ & Fault 2 (0, 2) & Fault 3 (0, 3) \\
\hline Kinds of spectra & 6 & 4 & 4 \\
Definition 1 & 6 & 3 & 5 \\
Definition 1 & 9 & 5 & 5 \\
Slice of bispectra & 7 & 9 & 2 \\
\hline
\end{tabular}

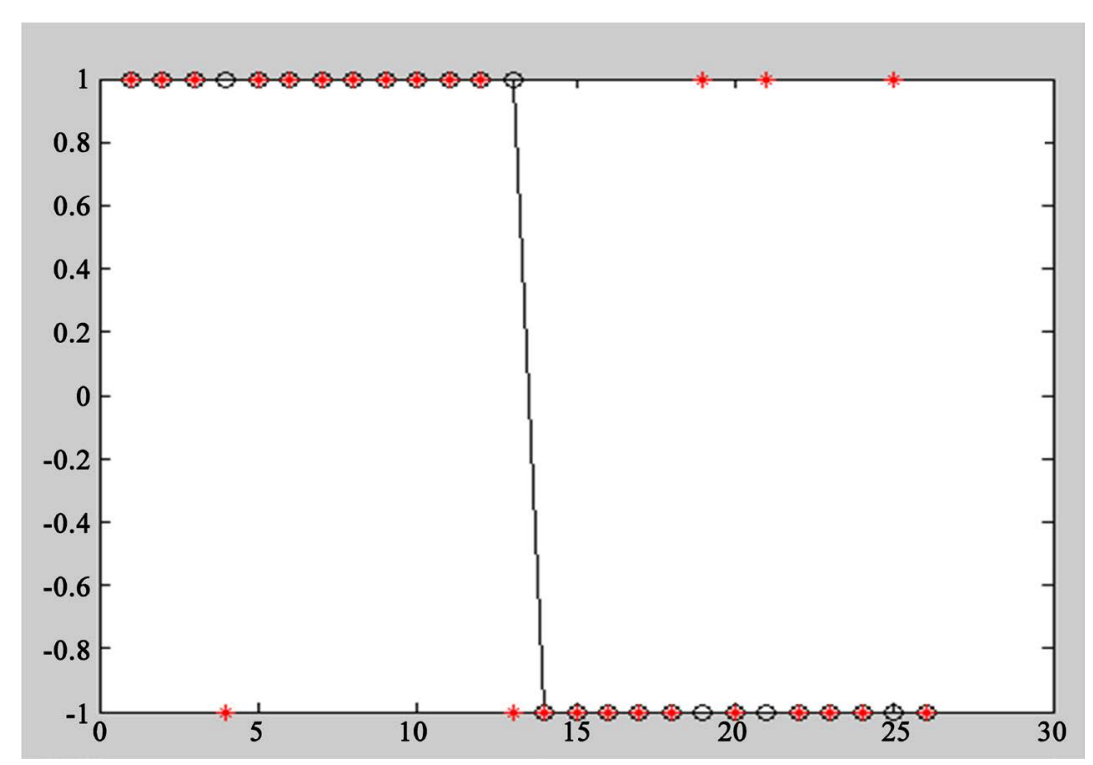

Figure 6. Simulation results of fault 3 of bispectral slice. 
and outlet of the pressure reducing valve. The oil pressure is constantly changing when data is collected, but the nature of the fault is inherent Features, such as failure three compared with failure two, is that an additional iron core is added to the oil inlet. The different characteristics of this different failure make it a certain essential difference between the normal signal. For the third fault, if the components participating in the coupling of the normal signal and the fault signal are retained at the same time, and the coupled components are removed, the characteristic difference between the normal signal and the fault signal is the most obvious at this time, Therefore, in this way, a good fault discrimination effect can be achieved.

The situation of fault 2 is the same as this. For fault 1 , the results of the three definition methods are not too satisfactory (the best is $6 / 26$, about $80 \%$ ), whether there are other more suitable methods to distinguish this kind of failure, this is also that this article found a place worthy of further study. This article found a place worthy of further study. For comparison, this paper also shows the diagnosis results of the bispectral diagonal slices for the three fault states in Table 2. The feature extraction and fault recognition steps are the same as the above. It can be seen that for the three fault states, the bispectral slices neither can achieve very satisfactory results, which should be due to the failure of the bispectral slice to process the coupling information in the original signal.

\section{Conclusion}

Although the mechanical vibration signal is extremely complex, each type of fault has its own inherent characteristics. This inherent feature makes the most essential difference between each type of fault signal and the normal signal. This article uses the third-order cumulative coupling to search for the nature of this difference, in the case of fault 2 and fault 3 , through different coupling methods, compared with the real bispectral diagonal slice, the fault diagnosis accuracy rate is improved to a satisfactory level. A result shows that there may be a certain specific difference between a specific mechanical fault and a normal signal. The most obvious information is extracted. This information will help improve the fault diagnosis rate, but at the same time it is found that this method is not suitable for each failure, and therefore, continuing to find other methods for each specific failure is a place worthy of further discussion in this article.

\section{Acknowledgements}

This paper is supported by Research Foundation of the Nanchang Normal University for Doctors (NSBSJJ2018014). Key R\&D Project of Jiangxi Provincial Department of Science and Technology (20192BBEL50040, 20192BBHL80002).

\section{Conflicts of Interest}

The authors declare no conflicts of interest regarding the publication of this paper. 


\section{References}

[1] Cai, Q.Z. and Huang, Y.J. (2009) Research on Autoregressive Trispectral Slices. Chinese Journal of Scientific Instrument, 30.

[2] Yang, J.G. and Xu, J.N. (2000) Characteristics and Identification of Dual Coherence Spectrum of Rotating Machinery Faults. Chinese Journal of Mechanical Engineering, 36,103-106.

[3] Wu, Z.G., Xia, L. and Yin, W.M. (2002) Modern Signal Processing Technology: High-Order Spectrum, Time-Frequency Analysis and Wavelet Transform. Wuhan University Press, Wuhan, 21-22.

[4] Hinich, M.J. (1994) Higher-Order Cumulants and Cumulants Spectra. Circuits Systems and Signal Processing, 13, 391-420. https://doi.org/10.1007/BF01183737

[5] Raughveer, M.R. (1990) Time-Domain Approaches to Quadratic Phase Coupling Estimation. IEEE Transactions on Automatic Control, 35,48-56. https://doi.org/10.1109/9.45142

[6] Zhang, Y. and Wang, S.X. (1998) Nonlinear Phase Coupling Slice Spectrum Analysis Method. Acta Electronica Sinica, 26, 104-109.

[7] Zhang, Y., Wang, S.X. and Li, S.H. (1996) 11/2 Dimensional Spectrum Analysis of Secondary Phase Coupling. Acta Electronics Sinica, 64, 109-112. 